Honam Mathematical J. 34 (2012), No. 2, pp. 161-169

http://dx.doi.org/10.5831/HMJ.2012.34.2.161

\title{
MINIMAXNESS OF LOCAL COHOMOLOGY MODULES DEFINED BY A PAIR OF IDEALS
}

\author{
A. Abbasi and H. Roshan Shekalgourabi
}

\begin{abstract}
Let $R$ be a commutative Noetherian ring and $I, J$ be ideals of $R$. We introduced the notion of $(I, J)$-cominimax $R$ modules. For an integer $n$ and an $R$-module $M$, let $H_{I, J}^{i}(M)$ be an $(I, J)$-cominimax $R$-module for all $i<n$. The $J$-minimaxness of some Ext modules of $H_{I, J}^{n}(M)$ is investigated. Among of the obtaining results, there is a generalization of the main result of [1].
\end{abstract}

\section{INTRODUCTION}

Throughout this paper, $R$ is a commutative Noetherian ring with identity and all modules are assumed to be unitary. Also, we assume that $I$ and $J$ are ideals of $R$. The generalized local cohomology module with respect to a pair of ideals $(I, J)$ of $R$ was introduced in [6]. Consider the subset

$$
W(I, J)=\left\{\mathfrak{p} \in \operatorname{Spec}(R) \mid I^{n} \subseteq \mathfrak{p}+J, \text { for an integer } n \geq 1\right\}
$$

of $\operatorname{Spec}(R)$. Recall that for an ideal $I$ of $R$ the Zariski closed set $V(I)$ is the set of all prime ideals of $R$ containing $I$. It is easy to see that $V(I) \subseteq W(I, J)$ and if $J=0$, then $W(I, J)$ coincides with the $V(I)$. Note that $W(I, J)$ is stable under specialization, but in general, it is not a closed subset of $\operatorname{Spec}(R)$. For an $R$-module $M,(I, J)$-torsion submodule of $M$ is the set of all elements $x$ of $M$ such that $I^{n} x \subseteq$ $J x$ for some positive integer $n$ and is denoted by $\Gamma_{I, J}(M)$. For any $R$-homomorphism $f: M \rightarrow N$ one has $f\left(\Gamma_{I, J}(M)\right) \subseteq \Gamma_{I, J}(N)$ and so $\Gamma_{I, J}(f)=\left.f\right|_{\Gamma_{I, J}(M)}$ is an $R$-homomorphism. Hence $\Gamma_{I, J}(-)$ is a covariant, $R$-linear functor from the category of $R$-modules to itself and in the case when $J=0,(I, J)$-torsion functor $\Gamma_{I, J}(-)$ is coincides with

Received October 26, 2011. Accepted December 6, 2011.

2000 Mathematics Subject Classification. 13D45, 13E10, $13 \mathrm{C} 05$.

Key words and phrases. Local cohomology modules, $J$-minimax modules, $(I, J)$ cominimax modules. 
the $I$-torsion functor $\Gamma_{I}(-)$. This functor is left exact and for an integer $i$, the local cohomology functor $H_{I, J}^{i}(-)$ with respect to $(I, J)$ is defined as the $i$-th right derived functor of $\Gamma_{I, J}(-)$ (see [6]). For an $R$-module $M, H_{I, J}^{i}(M)$ is called the $i$-th local cohomology module of $M$ with respect to $(I, J)$. It is obvious that if $J=0$, then $H_{I, J}^{i}(-)$ coincides with the ordinary local cohomology functor $H_{I}^{i}(-)$. Let $\widetilde{W}(I, J)$ to be the set of all ideals $\mathfrak{a}$ of $R$ such that $I^{n} \subseteq \mathfrak{a}+J$ for some integer $n$. For all $\mathfrak{a}, \mathfrak{b} \in \widetilde{W}(I, J)$ we define $\mathfrak{a} \leq \mathfrak{b}$ if $\mathfrak{a} \supseteq \mathfrak{b}$. So, $\widetilde{W}(I, J)$ is a partially ordered set. It is easy to see that $\widetilde{W}(I, J)$ is a system of ideals of $R$. Considering $\Phi=\widetilde{W}(I, J)$ one has $\Gamma_{I, J}(M)=\Gamma_{\Phi}(M)$. Hence $H_{I, J}^{i}(M)=H_{\Phi}^{i}(M)$ for all integers $i$, which studied by Bijan-Zadeh in [2].

For more informations of local cohomology module with respect to a pair of ideals $(I, J)$, we refer the reader to [6].

Recall that for an $R$-module $M$, the Goldie dimension of $M$ is defined as the cardinal of the set of indecomposable submodules of $E(M)$ which appear in a decomposition of $E(M)$ into a direct sum of indecomposable submodules. We use $G \operatorname{dim} M$ to denote Goldie dimension of $M$ (see [8]). For a prime ideal $\mathfrak{p}$ of $R$, let $\mu^{0}(\mathfrak{p}, M)$ denotes the 0 -th Bass number of $M$ with respect to the prime ideal $\mathfrak{p}$. So, it is obvious by definition of the Goldie dimension that

$$
G \operatorname{dim} M=\sum_{\mathfrak{p} \in \operatorname{Ass}_{R} M} \mu^{0}(\mathfrak{p}, M) .
$$

Also, for any ideal $I$ of $R$, the $I$-relative Goldie dimension of $M$, which is introduced in [3], is defined as

$$
G \operatorname{dim}_{I} M:=\sum_{\mathfrak{p} \in V(I)} \mu^{0}(\mathfrak{p}, M) .
$$

An $R$-module $M$ is called minimax if there is a finite submodule $N$ of $M$ such that $M / N$ is an Artinian $R$-module. It is shown in [10] that when $R$ is a Noetherian ring, an $R$-module $M$ is minimax if and only if for any submodule $N$ of $M, G \operatorname{dim} M / N<\infty$. An $R$-module $M$ is said to be $I$-minimax if for any submodule $N$ of $M, G \operatorname{dim}_{I} M / N<\infty$.

An $R$-module $M$ is $I$-cominimax if $\operatorname{Supp}(M) \subseteq V(I)$ and $\operatorname{Ext}_{R}^{i}(R / I, M)$ is a minimax $R$-module, for every $i \geq 0$ (see [1]). As a generalization of the concept of $I$-cominimax, we define the concept of $(I, J)$-cominimax module ( see Definition 2.1).

In this paper, among other results we will study $J$-minimaxness of $\operatorname{Ext}_{R}^{j}\left(R / I, H_{I, J}^{i}(M)\right)$, for $j=0,1,2$. More precisely, we show the followings hold. 
Theorem 1.1. Let $M$ be an $R$-module and $n$ be a non-negative integer such that $H_{I, J}^{i}(M)$ is $(I, J)$-cominimax, for all $i<n$ and $\operatorname{Ext}_{R}^{n}(R / I, M)$ is $J$-minimax. Then for any submodule $N$ of $H_{I, J}^{n}(M)$ where $\operatorname{Ext}_{R}^{1}(R / I, N)$ is $J$-minimax and for any finite $R$-module $T$ with Supp $T \subseteq V(I)$, the $R$-module $\operatorname{Hom}_{R}\left(T, H_{I, J}^{n}(M) / N\right)$ is $J$-minimax.

Theorem 1.2. Let $M$ be an R-module and $n$ be a non-negative integer such that $H_{I, J}^{i}(M)$ is $(I, J)$-cominimax, for all $i<n$. If $\operatorname{Ext}_{R}^{n+1}(R / I, M)$ is a $J$-minimax $R$-module, then $\operatorname{Ext}_{R}^{1}\left(R / I, H_{I, J}^{n}(M)\right)$ is $J$-minimax.

Theorem 1.3. Let $M$ be an $R$-module and $n$ be a non-negative integer such that $H_{I, J}^{i}(M)$ is $(I, J)$-cominimax, for all $i<n$. If $\operatorname{Ext}_{R}^{i}(R / I, M)$ is $J$ minimax , for all $i \geq 0$, then $\operatorname{Hom}_{R}\left(R / I, H_{I, J}^{n+1}(M)\right)$ is $J$-minimax if and only if $\operatorname{Ext}_{R}^{2}\left(R / I, H_{I, J}^{n}(M)\right)$ is $J$-minimax.

\section{MAIN RESULTS}

We begin this section by introducing a new class of modules. Our motivation is the paper [1] in which the notion $I$-cominimax was introduced.

Definitions 2.1. An R-module $M$ is said to be $(I, J)$-cominimax if $\operatorname{Supp}(M) \subseteq W(I, J)$ and $\operatorname{Ext}_{R}^{i}(R / I, M)$ is a $J$-minimax $R$-module, for every $i \geq 0$.

Example 2.2. Let $I, J$ be ideals of $R$ and $M$ be an $R$-module. It is clear that any $J$-minimax module with support in $W(I, J)$ is a $(I, J)$ cominimax $R$-module. In particular, any minimax $R$-module with support in $W(I, J)$ is $(I, J)$-cominimax. Also, if $J=0$, then $M$ is $(I, J)$ cominimax if and only if $M$ is an $I$-cominimax $R$-module.

Remark 2.3. By [1, Proposition 2.3], the class of J-minimax modules is closed under submodules, quotients and taking extensions, i.e. it is a Serre subcategory of all $R$-modules. So, for $R$-modules $M$ and $N$ which are finite and $J$-minimax respectively, one has $\operatorname{Ext}_{R}^{i}(M, N)$ and $\operatorname{Tot}_{i}^{R}(M, N)$ are $J$-minimax for all $i \geq 0$.

The following lemma plays a key role in what follow:

Lemma 2.4. Let $M$ be an $(I, J)$-torsion-free $R$-module i.e. $\Gamma_{I, J}(M)=$ 0 and $E$ be the injective hull of $M$. If $L=E / M$, then $\operatorname{Ext}_{R}^{i-1}(R / I, L) \cong$ $\operatorname{Ext}_{R}^{i}(R / I, M)$ and $H_{I, J}^{i-1}(L) \cong H_{I, J}^{i}(M)$, for all $i>0$.

Proof. See [7, Remark 2.2]. 
Proposition 2.5. Let $n$ be an integer. If $M$ is an $R$-module such that $H_{I, J}^{i}(M)$ is $(I, J)$-cominimax for all $i \leq n$ (respectively for all $i$ ), then $\operatorname{Ext}_{R}^{i}(R / I, M)$ is $J$-minimax for all $i \leq n$ (respectively for all $i$ ).

Proof. We use induction on $n$. If $n=0$, since $H_{I, J}^{0}(M)=\Gamma_{I, J}(M)$ is $(I, J)$-cominimax and $\operatorname{Hom}(R / I, M) \cong \operatorname{Hom}_{R}\left(R / I, \Gamma_{I, J}(M)\right)$, the result holds.

Now, suppose that $n>0$ and the case $n-1$ is settled. Since $\Gamma_{I, J}(M)$ is $(I, J)$-cominimax, $\operatorname{Ext}_{R}^{j}\left(R / I, \Gamma_{I, J}(M)\right)$ is $J$-minimax, for every $j \geq 0$. By using the exact sequence

$$
0 \rightarrow \Gamma_{I, J}(M) \rightarrow M \rightarrow M / \Gamma_{I, J}(M) \rightarrow 0
$$

it is enough to show that $\operatorname{Ext}_{R}^{j}\left(R / I, M / \Gamma_{I, J}(M)\right)$ is $J$-minimax, for all $j \leq n$. We show that $\operatorname{Ext}_{R}^{j}(R / I, M)$ is $J$-minimax for all $(I, J)$-torsionfree $R$-modules $M$ and all $j \leq n$. Let $E$ be the injective hull of $M$ and let $L=E / M$. Now, by Lemma $2.4, H_{I, J}^{i}(L)$ is $(I, J)$-cominimax, for every $i \leq n-1$. Thus, by induction hypothesis, $\operatorname{Ext}_{R}^{i}(R / I, L)$ is a $J$-minimax $R$-module, for every $i \leq n-1$. Therefore, $\operatorname{Ext}_{R}^{i}(R / I, M)$ is $J$-minimax, for all $i \leq n$.

Proposition 2.6. Let $M$ be an $R$-module such that $\operatorname{Ext}_{R}^{i}(R / I, M)$ is $J$-minimax, for all $i$. If $n$ is a non-negative integer such that $H_{I, J}^{i}(M)$ is $(I, J)$-cominimax, for all $i \neq n$, then $H_{I, J}^{n}(M)$ is $(I, J)$-cominimax.

Proof. We use induction on $n$. Put $\bar{M}=M / \Gamma_{I, J}(M)$. The exact sequence $0 \rightarrow \Gamma_{I, J}(M) \rightarrow M \rightarrow \bar{M} \rightarrow 0$ induces the long exact sequence $\cdots \rightarrow \operatorname{Ext}_{R}^{i-1}(R / I, \bar{M}) \rightarrow \operatorname{Ext}_{R}^{i}\left(R / I, \Gamma_{I, J}(M)\right) \rightarrow \operatorname{Ext}_{R}^{i}(R / I, M)$

$$
\rightarrow \operatorname{Ext}_{R}^{i}(R / I, \bar{M}) \rightarrow \operatorname{Ext}_{R}^{i+1}\left(R / I, \Gamma_{I, J}(M)\right) \rightarrow \operatorname{Ext}_{R}^{i+1}(R / I, M) \rightarrow \cdots(*)
$$

for all $i \geq 0$. It also implies that $H_{I, J}^{i}(M) \cong H_{I, J}^{i}(\bar{M})$, for all $i \geq 1$. If $n=0, H_{I, J}^{i}(\bar{M})$ is $(I, J)$-cominimax, for all $i$ (note that $H_{I, J}^{0}(\bar{M})=0$ ). Hence, by Proposition 2.5, $\operatorname{Ext}_{R}^{i}(R / I, \bar{M})$ is $J$-minimax, for all $i$. So, by the exact sequence $(*)$ it follows that $H_{I, J}^{0}(M)$ is $(I, J)$-cominimax. Now, assume that $n>0$ and the assertion has been proved for $n-1$. Since, $H_{I, J}^{0}(M)$ is $(I, J)$-cominimax, by the exact sequence $(*)$ we may assume that $\Gamma_{I, J}(M)=0$. Let $E$ be the injective hull of $M$ and put $L=E / M$. Now, the assertion holds by Lemma 2.4 and induction hypothesis.

Corollary 2.7. Let $I$ be a principal ideal of $R$ and $M$ be a $J$-minimax $R$-module. Then $H_{I, J}^{i}(M)$ is $(I, J)$-cominimax, for all $i \geq 0$. 
Proof. Put $\bar{R}=R /\left(J+\operatorname{Ann}_{R} M\right)$. Then $I \bar{R}$ is a principal ideal of $\bar{R}$. So, $H_{I, J}^{i}(M)=0$, for all $i>1$ by [6, Theorem 2.7 and Proposition 4.10]. Also, $\Gamma_{I, J}(M)$ is $(I, J)$-cominimax by Remark 2.3. So, the assertion holds by Proposition 2.6.

The following theorem is a generalization of the main result of [1].

Theorem 2.8. Let $M$ be an $R$-module and $n$ be a non-negative integer such that $H_{I, J}^{i}(M)$ is $(I, J)$-cominimax, for all $i<n$ and $\operatorname{Ext}_{R}^{n}(R / I, M)$ is $J$ minimax. Then for any submodule $N$ of $H_{I, J}^{n}(M)$ such that $\operatorname{Ext}_{R}^{1}(R / I, N)$ is $J$ minimax and for any finite $R$-module $T$ with Supp $T \subseteq V(I), \operatorname{Hom}_{R}\left(T, H_{I, J}^{n}(M)\right.$ $(N)$ is a $J$-minimax $R$-mdoule.

Proof. By Gruson's theorem [9, Theorem 4.1] we have to prove the theorem for $T=R / I$. Also, by the long exact sequence

$\operatorname{Hom}\left(R / I, H_{I, J}^{t}(M)\right) \rightarrow \operatorname{Hom}\left(R / I, H_{I, J}^{t}(M) / N\right) \rightarrow \operatorname{Ext}_{R}^{1}(R / I, N) \rightarrow \cdots$

it is enough to show that $\operatorname{Hom}_{R}\left(R / I, H_{I, J}^{n}(M)\right)$ is $J$-minimax. One can argue by induction on $n$. Since $\operatorname{Hom}(R / I, M) \cong \operatorname{Hom}_{R}\left(R / I, \Gamma_{I, J}(M)\right)$ the assertion is clear for $n=0$. Now, suppose that $n>0$ and that the claim has been proved for $n-1$. Since $\Gamma_{I, J}(M)$ is an $(I, J)$-cominimax $R$-module, $\operatorname{Ext}_{R}^{i}\left(R / I, \Gamma_{I, J}(M)\right)$ is $J$-minimax, for all $i \geq 0$. So, by the long exact sequence

$$
\cdots \rightarrow \operatorname{Ext}_{R}^{n}(R / I, M) \rightarrow \operatorname{Ext}_{R}^{n}\left(R / I, M / \Gamma_{I, J}(M)\right) \rightarrow \operatorname{Ext}_{R}^{n+1}\left(R / I, \Gamma_{I, J}(M)\right)
$$

and Remark 2.3, $\operatorname{Ext}_{R}^{n}\left(R / I, M / \Gamma_{I, J}(M)\right)$ is $J$-minimax. Hence, we may assume that $M$ is $(I, J)$-torsion-free. Let $E$ be the injective hull of $M$ and let $L=E / M$. Now, the result holds by Lemma 2.4 and induction hypothesis.

Corollary 2.9. Let $M$ be a $J$-minimax $R$-module and let $n$ be a non-negative integer such that $H_{I, J}^{i}(M)$ is $(I, J)$-cominimax, for all $i<n$. If $J H_{I, J}^{n}(M)$ is a $J$-minimax $R$-module, then $H_{I, J}^{n}(M) / J H_{I, J}^{n}(M)$ has finite Goldie dimension. In particular, the set of associated primes of $H_{I, J}^{n}(M) / J H_{I, J}^{n}(M)$ is finite.

Proof. By Theorem 2.8, $\operatorname{Hom}_{R}\left(R / I, H_{I, J}^{n}(M) / J H_{I, J}^{n}(M)\right)$ is a $J$-mini $\max R$-module. By [6, Corollary 1.9], $H_{I, J}^{n}(M) / J H_{I, J}^{n}(M)$ is an $I$ torsion module. So, we obtain that

$$
\operatorname{Ass}_{R} \operatorname{Hom}_{R}\left(R / I, H_{I, J}^{n}(M) / J H_{I, J}^{n}(M)\right)=\operatorname{Ass}_{R} H_{I, J}^{n}(M) / J H_{I, J}^{n}(M) .
$$

Now, the result follows by [1, Corollary 3.6]. 
Corollary 2.10. Let $M$ be an $R$-module and $n \geq 1$ be an integer such that $\operatorname{Ext}_{R}^{n-1}(R / I, M)$ be a finite $R$-module and $\operatorname{Supp} H_{I, J}^{i}(M) \subseteq$ $\operatorname{Max}(R)$, for all $i<n$. If $\operatorname{Ext}_{R}^{1}\left(R / I, J H_{I, J}^{i}(M)\right)$ is finite, for all $i<n$, then $H_{I, J}^{n}(M) / J H_{I, J}^{n}(M)$ is Artinian, for all $i<n$.

Proof. We argue by induction on $n$. If $n=1$, then $\left(0:_{\Gamma_{I, J}(M)} I\right)$ is Artinian, since it is a finite $R$-module with support in $\operatorname{Max}(R)$. Consider the exact sequence

$$
0 \rightarrow J \Gamma_{I, J}(M) \rightarrow \Gamma_{I, J}(M) \rightarrow \Gamma_{I, J}(M) / J \Gamma_{I, J}(M) \rightarrow 0 .
$$

Then $\operatorname{Ext}_{R}^{1}\left(R / I, J \Gamma_{I, J}(M)\right)$ is Artinian, since it is a finite $R$-module with support in $\operatorname{Max}(R)$. So, $\operatorname{Hom}\left(R / I, \Gamma_{I, J}(M) / J \Gamma_{I, J}(M)\right)$ is Artinian. As $\Gamma_{I, J}(M) / J \Gamma_{I, J}(M)$ is $I$-torsion, it yields from [4, Theorem 1.3] that $\Gamma_{I, J}(M) / J \Gamma_{I, J}(M)$ is Artinian. Assume that $n \geq 2$ and the assertion holds for $n-1$. Since, $H_{I, J}^{i}(M) \cong H_{I, J}^{i}\left(M / \Gamma_{I, J}(M)\right)$, for all $i \geq 1$ and $\Gamma_{I, J}\left(M / \Gamma_{I, J}(M)\right)=0$ we may assume that $\Gamma_{I, J}(M)=0$. Now, by a similar proof used in Theorem 2.8, the inductive step is complete.

An $R$-module $M$ is called $(I, J)$-cofinite if $\operatorname{Supp}(M) \subseteq W(I, J)$ and $\operatorname{Ext}_{R}^{i}(R / I, M)$ is a finite $R$-module, for every $i \geq 0$ (see [7]).

Lemma 2.11. If $M$ is a $J$-minimax $R$-module with $\operatorname{Supp} M \subseteq W(I, J)$ and if $J M$ is an $I$-cofinite $R$-module, then $M$ is an $(I, J)$-cofinite $R$ module if and only if $\left(0:_{M} I\right)$ is a finite $R$-module.

Proof. By [6, Proposition 1.7 and Corollary 1.9] we have $M / J M$ is an $I$-torsion $R$-module. On the other hand, $M / J M$ is an $J$-torsion $R$ module and so is minimax by assumption. Hence, by [5, Proposition 4.3], $M / J M$ is $I$-cofinite if and only if $\left(0:_{M / J M} I\right)$ is finite. Now, the result holds by the following exact sequence:

$$
0 \rightarrow J M \rightarrow M \rightarrow M / J M \rightarrow 0 .
$$

Proposition 2.12. Let $M$ be a finite $R$-module and $n$ be a nonnegative integer such that $H_{I, J}^{i}(M)$ is $J$-minimax, for all $i<n$. If $J H_{I, J}^{i}(M)$ is finite, for all $i<n$, then $\operatorname{Hom}_{R}\left(R / I, H_{I, J}^{n}(M)\right)$ is finite.

Proof. We proceed by induction on $n$. The case $n=0$ is obvious since $H_{I, J}^{0}(M)$ is finite. So, let $n>0$, and the result has been proved for smaller values of $n$. By the induction hypothesis, $\operatorname{Hom}_{R}\left(R / I, H_{I, J}^{i}(M)\right)$ is finite $R$-module for all $i<n$. Therefore, by Lemma 2.11, $H_{I, J}^{i}(M)$ 
is $(I, J)$-cofinite for all $i<n$. Now, the result follows from [7, Theorem 2.3].

Theorem 2.13. Let $M$ be an $R$-module and $n$ be a non-negative integer such that $H_{I, J}^{i}(M)$ is $(I, J)$-cominimax, for all $i<n$. If $\operatorname{Ext}_{R}^{n+1}(R / I, M)$ is a $J$-minimax $R$-module, then $\operatorname{Ext}_{R}^{1}\left(R / I, H_{I, J}^{n}(M)\right)$ is $J$-minimax.

Proof. We use induction on $n$. If $n=0$, then $\operatorname{Ext}_{R}^{1}(R / I, M)$ is a $J$ minimax $R$-module. Put $\bar{M}=M / \Gamma_{I, J}(M)$. So, by the exact sequence

$$
0 \rightarrow \Gamma_{I, J}(M) \rightarrow M \rightarrow \bar{M} \rightarrow 0
$$

and Remark 2.3 it follows that $\operatorname{Ext}_{R}^{1}\left(R / I, \Gamma_{I, J}(M)\right)$ is $J$-minimax. Now, suppose that $n>0$ and the result is true for $n-1$. Since $\Gamma_{I, J}(M)$ is $(I, J)$-cominimax, the $R$-module $\operatorname{Ext}_{R}^{i}\left(R / I, \Gamma_{I, J}(M)\right)$ is $J$-minimax, for all $i$ and so $\operatorname{Ext}_{R}^{n+1}(R / I, \bar{M})$ is $J$-minimax. Therefore, we may assume that $\left.\Gamma_{I, J}(M)\right)=0$. Hence by Lemma 2.4 and the induction hypothesis,

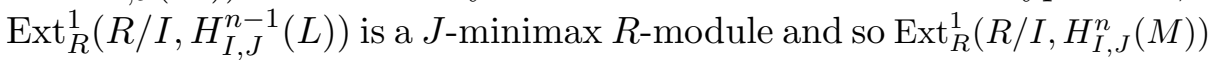
is $J$-minimax.

Corollary 2.14. Let $M$ be an $R$-module with injective dimension $n \geq 0$. If $H_{I, J}^{i}(M)$ is $(I, J)$-cominimax, for all $i<n$, then $\operatorname{Ext}_{R}^{1}\left(R / I, H_{I, J}^{n}(M)\right)$ is $J$-minimax.

Theorem 2.15. Let $M$ be an $R$-module and $n$ be a non-negative integer such that $H_{I, J}^{i}(M)$ is $(I, J)$-cominimax, for all $i<n$. If $\operatorname{Ext}_{R}^{i}(R / I, M)$ is $J$-minimax, for all $i \geq 0$, then $\operatorname{Hom}_{R}\left(R / I, H_{I, J}^{n+1}(M)\right)$ is $J$-minimax if and only if $\operatorname{Ext}_{R}^{2}\left(R / I, H_{I, J}^{n}(M)\right)$ is $J$-minimax.

Proof. Put $\bar{M}=M / \Gamma_{I, J}(M)$ and $L=E / \bar{M}$ which $E$ is the injective hull of $\bar{M}$.

$\Rightarrow$ ) We proceed by induction on $n$. Let $n=0$. Then, by the long exact sequence

$$
\cdots \rightarrow \operatorname{Ext}_{R}^{1}(R / I, \bar{M}) \rightarrow \operatorname{Ext}_{R}^{2}\left(R / I, \Gamma_{I, J}(M)\right) \rightarrow \operatorname{Ext}_{R}^{2}(R / I, M) \rightarrow \cdots
$$

it is enough to show that $\operatorname{Ext}_{R}^{1}(R / I, \bar{M})$ is $J$-minimax. By Lemma 2.4, we conclude that

$$
\begin{aligned}
\operatorname{Ext}_{R}^{1}(R / I, \bar{M}) & \cong \operatorname{Hom}_{R}(R / I, L) \\
& \cong \operatorname{Hom}_{R}\left(R / I, \Gamma_{I, J}(L)\right) \\
& \cong \operatorname{Hom}_{R}\left(R / I, H_{I, J}^{1}(M)\right) .
\end{aligned}
$$

Now, suppose that $n>0$ and the case $n-1$ is settled. Since $\Gamma_{I, J}(M)$ is $(I, J)$-cominimax, the $R$-module $\operatorname{Ext}_{R}^{i}\left(R / I, \Gamma_{I, J}(M)\right)$ is $J$-minimax, for 
all $i$. Using the exact sequence

$\cdots \rightarrow \operatorname{Ext}_{R}^{i}(R / I, M) \rightarrow \operatorname{Ext}_{R}^{i}(R / I, \bar{M}) \rightarrow \operatorname{Ext}_{R}^{i+1}\left(R / I, \Gamma_{I, J}(M)\right) \rightarrow \cdots$,

we observe that $\operatorname{Ext}_{R}^{i}(R / I, \bar{M})$ is $J$-minimax, for every $i$. So, $\operatorname{Ext}_{R}^{i}(R / I, L)$ is a $J$-minimax $R$-module, for all $i$.

Also $\operatorname{Hom}_{R}\left(R / I, H_{I, J}^{n}(L)\right) \cong \operatorname{Hom}_{R}\left(R / I, H_{I, J}^{n+1}(M)\right)$ is a $J$-minimax $R$ module. Now, by the induction hypothesis which is replaced $M$ by $L$, we obtain that $\operatorname{Ext}_{R}^{2}\left(R / I, H_{I, J}^{n-1}(L)\right)$ is a $J$-minimax $R$-module. Therefore, it follows that $\operatorname{Ext}_{R}^{2}\left(R / I, H_{I, J}^{n}(M)\right)$ is $J$-minimax.

$\Leftarrow)$ Using the induction on $n$, let $n=0$. By the exact sequence $\cdots \rightarrow \operatorname{Ext}_{R}^{1}(R / I, M) \rightarrow \operatorname{Ext}_{R}^{1}(R / I, \bar{M}) \rightarrow \operatorname{Ext}_{R}^{2}\left(R / I, \Gamma_{I, J}(M)\right) \rightarrow \cdots$, it follows that $\operatorname{Ext}_{R}^{1}(R / I, \bar{M})$ is $J$-minimax. Hence, $\operatorname{Hom}_{R}(R / I, L)$ is a $J$-minimax $R$-module and so is $\operatorname{Hom}_{R}\left(R / I, \Gamma_{I, J}(L)\right)$.

Thus, $\operatorname{Hom}_{R}\left(R / I, H_{I, J}^{1}(M)\right)$ is a $J$-minimax $R$-module. Now, suppose that $n>0$. We observe that the $R$-modules $\operatorname{Ext}_{R}^{2}\left(R / I, H_{I, J}^{n-1}(L)\right)$ and $\operatorname{Ext}_{R}^{i}(R / I, L)$ are $J$-minimax, for all $i$. Now, the induction hypothesis follows that the $R$-module

$$
\operatorname{Hom}_{R}\left(R / I, H_{I, J}^{n}(L)\right) \cong \operatorname{Hom}_{R}\left(R / I, H_{I, J}^{n+1}(M)\right)
$$

is $J$-minimax.

Corollary 2.16. Let $M$ be a $J$-minimax $R$-module and let $n$ be a non-negative integer such that $H_{I, J}^{i}(M)$ is $(I, J)$-cominimax, for all $i<n$.

Then $\operatorname{Hom}_{R}\left(R / I, H_{I, J}^{n+1}(M)\right)$ is $J$-minimax if and only if $\operatorname{Ext}_{R}^{2}\left(R / I, H_{I, J}^{n}\right.$ $(M))$ is $J$-minimax.

\section{References}

[1] J. Azami, R. Naghipour, and B. Vakili, Finiteness properties of local cohomology modules for a-minimax modules, Proc. Amer. Math. Soc. 137 (2009), no. 2, 439-448.

[2] M. H. Bijan-Zadeh, A common generalization of local cohomology theories, Glasgow Math. J. 21 (1980), no. 2, 173-181.

[3] K. Divaani-Aazar and M. Esmkhani, Artinianness of local cohomology modules of ZD-modules, Comm. Algebra 33 (2005), 2857-2863.

[4] L. Melkersson, On asymptotic stability for sets of prime ideals connected with the powers of an ideal, Math. Proc. Cambridge Philos. Soc. 107 (1990), 267-271.

[5] _ Modules cofinite with respect to an ideal, J. Algebra 285 (2005), 649-668.

[6] R. Takahashi, Y. Yoshino, and T. Yoshizawa, Local cohomology based on a nonclosed support defined by a pair of ideals, J. Pure Appl. Algebra 213 (2009), no. $4,582-600$. 
[7] A. Tehranian and A. Pour Eshmanan Talemi, Cofiniteness of local cohomology based on a nonclosed support defined by a pair of ideals, Bull. Iranian. Math. Soc. 36 (2010), no. 2, 145-155.

[8] P. Vamos and D.W. Sharpe, Injective modules, Cambridge University Press, London-New York, 1972.

[9] W. Vasconcelos, Divisor theory in module categories, North-Holland Publishing Company,Amsterdam, 1974.

[10] H. Zöschinger, Minimax-moduln, J. Algebra 102 (1986), 1-32.

\section{A. Abbasi}

Department of Pure Mathematics, University of Guilan, P.O. Box 41335-19141, Rasht, Iran.

E-mail: aabbasi@guilan.ac.ir

H. Roshan Shekalgourabi

Department of Pure Mathematics, University of Guilan, P.O. Box 41335-19141, Rasht, Iran.

E-mail: hroshan@guilan.ac.ir 